\title{
Incidence and Determinants of QT Interval Prolongation in COVID-19 Patients Treated with Hydroxychloroquine and Azithromycin
}

\author{
Ilir Maraj ${ }^{1}$, James Hummel ${ }^{1}$, Roy Taoutel $^{1}$, Romy Chamoun ${ }^{1}$, Virginia Workman ${ }^{1}$, Cindy \\ $\mathrm{Li}^{2}$, Lydia Tran ${ }^{2}$, Alexander DelVecchio ${ }^{3}$, Christopher Howes ${ }^{1}$, and joseph akar ${ }^{1}$ \\ ${ }^{1}$ Yale University School of Medicine \\ ${ }^{2}$ Yale New Haven Health System \\ ${ }^{3}$ Greenwich Hospital
}

May 26, 2020

\begin{abstract}
Combined use of hydroxychloroquine and azithromycin was globally adopted, in part due to paucity and high cost of alternative therapies. However the utility of these medications has been questioned; and thus safety becomes a major concern given clinical equipoise regarding efficacy. Both hydroxychloroquine and azithromycin continue to be administered in US clinical trials examining their potential role in prevention of infection, treatment of mild infection in ambulatory patients, and in combination with other medical regimens in treatment of patients with severe disease. These drugs also continue to be clinically utilized in hospitalized patients around the globe, often without continuous telemetry due to lack of resources. Concern regarding use of hydroxychloroquine without adequate rhythm monitoring in clinical trials has been recently expressed.1 A review of clinicaltrials.gov at the time of submission of this correspondence reveals actively recruiting trials of combined hydroxychloroquine/azithromycin with or without additional COVID-19 therapies, for both ambulatory and hospitalized patients within and outside the US. The potential for hydroxychloroquine and azithromycin to cause QT prolongation is counterbalanced by very low risk of pro-arrhythmia in the general population, and emerging evidence of relatively low risk of Torsades de Pointes (TdP) in COVID-19 patients.2,3,4,5 Thus delineation of the determinants of significant QTc prolongation and pro-arrhythmic risk for hydroxychloroquine/azithromycin is very important, especially given mounting evidence of inefficacy in COVID-19 treatment.
\end{abstract}

Incidence and Determinants of QT Interval Prolongation in COVID-19 Patients Treated with Hydroxychloroquine and Azithromycin

Ilir Maraj ${ }^{1,2^{*}}$, James P Hummel ${ }^{*}$, Roy Taoutel ${ }^{1}$, Romy Chamoun ${ }^{1}$, Virginia Workman ${ }^{1}$, Cindy Li $^{3}$, Lydia $\operatorname{Tran}^{3}$, Alexander DelVecchio ${ }^{2}$, Christopher Howes ${ }^{1,2}$, Joseph G Akar ${ }^{1,2}$

1. Department of Internal Medicine, Cardiovascular Medicine, Yale School of Medicine, New Haven, CT

2. Department of Internal Medicine, Greenwich Hospital, Greenwich, CT

3. Department of Pharmacy Services, Yale New Haven Hospital, New Haven, CT

*Authors contributed equally

\section{Word Count: 1091}

\section{Disclosures:}

No relationships with industry to be disclosed related to this specific work.

Joseph Akar reports consulting for Biosense Webster. 


\section{Address for Correspondence:}

Joseph G. Akar, MD, PhD

Section of Cardiovascular Medicine / Yale University School of Medicine

Dana 3, 789 Howard Ave

New Haven, CT 06520

Tel: 203-737-4716

Fax 203-785-6506

Email: joseph.akar@yale.edu

Combined use of hydroxychloroquine and azithromycin was globally adopted, in part due to paucity and high cost of alternative therapies. However the utility of these medications has been questioned; and thus safety becomes a major concern given clinical equipoise regarding efficacy. Both hydroxychloroquine and azithromycin continue to be administered in US clinical trials examining their potential role in prevention of infection, treatment of mild infection in ambulatory patients, and in combination with other medical regimens in treatment of patients with severe disease. These drugs also continue to be clinically utilized in hospitalized patients around the globe, often without continuous telemetry due to lack of resources. Concern regarding use of hydroxychloroquine without adequate rhythm monitoring in clinical trials has been recently expressed. ${ }^{1}$ A review of clinicaltrials.gov at the time of submission of this correspondence reveals actively recruiting trials of combined hydroxychloroquine/azithromycin with or without additional COVID-19 therapies, for both ambulatory and hospitalized patients within and outside the US. The potential for hydroxychloroquine and azithromycin to cause QT prolongation is counterbalanced by very low risk of pro-arrhythmia in the general population, and emerging evidence of relatively low risk of Torsades de Pointes (TdP) in COVID-19 patients. ${ }^{2,3,4,5}$ Thus delineation of the determinants of significant QTc prolongation and pro-arrhythmic risk for hydroxychloroquine/azithromycin is very important, especially given mounting evidence of inefficacy in COVID-19 treatment.

\section{Methods}

We conducted a retrospective analysis of consecutive symptomatic patients who were hospitalized for COVID19 infection and received treatment with combination hydroxychloroquine/azithromycin. This study was approved by the Yale University School of Medicine Human Investigation Committee. Baseline 12-lead ECGs were obtained prior to initiation of therapy. Patients underwent continuous telemetry and the corrected QT interval (QTc) was measured by an electrophysiologist at baseline and then daily using Bazett's formula. Independent variables of interest were those associated with QTc prolongation or disease severity (age, gender, baseline QTc, concurrent use of other high risk QT-prolonging medications, and laboratory markers of inflammation and infection (leukocytes, C-Reactive Protein), disease severity (Troponin T, D-Dimer), renal insufficiency (GFR $<60 \mathrm{ml} / \mathrm{min}$ ) and hypokalemia (serum potassium). Definition of high-risk QTc medications was based on the classification by the Arizona Center for Education and Research on Therapeutics (AZCERT). The primary outcome was the development of significant QTc prolongation defined as increase in baseline QTc [?]60 ms and/or absolute QTc > $500 \mathrm{~ms} .{ }^{6}$ Secondary outcomes included ventricular tachyarrhythmias (TdP, polymorphic VT or VF).

Demographic, clinical and laboratory characteristics were compared between patients who developed QTc prolongation compared to those who did not. Continuous variables are expressed as mean \pm standard deviation (SD). Proportions were compared using the $\chi^{2}$ test. Continuous variables were compared using the t-test for normally distributed data or the Mann-Whitney U test if non-normal. Statistical significance was defined as $\mathrm{p}<0.05$. Unadjusted odds ratios $(\mathrm{OR})$ were calculated separately for each potential predictor of significant QT prolongation using univariable logistic regression. Significant predictors $(\mathrm{p}<0.05)$ from univariable analysis were then used as covariates in a multiple logistic regression model to calculate adjusted odds ratios (aOR). 


\section{Results}

The cohort consisted of 91 patients (age $62.7 \pm 15.1$ years, $44 \%$ females,). Excessive QTc prolongation occurred in $23 \%$ of patients receiving hydroxychloroquine/azithromycin, increasing from $437 \pm 37$ to $504 \pm 41$ ms. In $14 \%$ of the cohort, the QTc exceeded $500 \mathrm{~ms}$. The baseline characteristics of those with and without significant prolongation are shown in Table 1. Patients with excessive prolongation were generally older (age $70.0 \pm 15.1$ vs $60.5 \pm 14.5, \mathrm{p}=0.016)$, with more hypertension $(58 \%$ vs $40 \%, \mathrm{p}=0.045)$, renal insufficiency $(52 \%$ vs $20 \%, \mathrm{p}=0.006)$, coronary artery ( $33 \%$ vs $8 \%, \mathrm{p}=0.01)$ and cerebrovascular disease $(24 \%$ vs $3 \%, \mathrm{p}=0.01)$. In addition they appeared to develop greater severity of disease with 10/21 (48\%) requiring mechanical ventilation compared to 15/70 (21\%) without significant QT prolongation ( $\mathrm{p}=0.03) .9 / 21(43 \%)$ patients with excessive QT prolongation had baseline renal insufficiency or developed acute renal failure. An additional concurrent QT-prolonging medication was administered in $42 \%$ of patients. Among patients with excessive QT prolongation, a concurrent QT prolonging drug was used in $67 \%$ of patients versus only $34 \%$ in patients without excessive QT prolongation ( $\mathrm{p}=0.01$ ). Most was due to intravenous propofol, which was used in $48 \%$ of patients with excessive QT prolongation compared to only $19 \%$ of patients without $(\mathrm{p}=0.01)$.

Significant ventricular arrhythmias occurred in 2 patients. One patient had classic TdP (Figure 1) and the second patient developed polymorphic VT that degenerated into VF in the setting of severe multisystem disease. Table 2 shows the results of the univariable and multiple logistic regression analysis. Older age $(>75$ years), prolonged baseline QTc ( $>460 \mathrm{~ms}$ ), impaired renal function (GFR $<60 \mathrm{ml} / \mathrm{min}$ ), and concurrent use of a high risk QTc-prolonging drug were each associated with excessive QTc prolongation. Multiple regression demonstrated the use of additional QT prolonging agents (especially propofol) to be independently associated with QTc prolongation [any drug: adjusted OR 3.69, CI $(1.22,11.20), \mathrm{p}=0.02$; propofol: adjusted OR 3.28, CI $(1.06,10.17), \mathrm{p}=0.04]$.

\section{Discussion}

We found that combined hydroxychloroquine/azithromycin results in significant QTc prolongation in approximately one in four hospitalized patients. The degree of QTc prolongation is severe, exceeding $500 \mathrm{~ms}$ in $14 \%$ of patients, and resulting in a case of TdP. Thus combined hydroxychloroquine/azithromycin should not be administered without continuous telemetry monitoring, even in the setting of a clinical trial. Determinants of QTc prolongation include older age, impaired renal function, baseline QTc $>460 \mathrm{~ms}$, and concurrent use of other high-risk QTc-prolonging drugs. Care is required to minimize the concurrent administration of these medications. Vigilance in QTc and arrhythmia monitoring is required for patients in whom the concurrent use of high-risk QTc drugs is necessary, with particular attention to intravenous propofol given the frequency of its use in severe infection. Propofol, which is often used as a sole agent without clinical sequalae, may increase pro-arrhythmic risk when administered with hydroxychloroquine/azithromycin in patients with severe COVID-19 infection.

\section{Limitations}

Although there is a high degree of collinearity between severity of illness, mechanical ventilation and propofol use, this only highlights the importance of considering the determinants of QTc prolongation in this very sick population.

\section{Conclusions}

Despite equipoise regarding the benefit of hydroxychloroquine/azithromycin treatment for COVID-19 infection, these medications continue to be used in large clinical trials both in the US and across the world. This study demonstrates that the risk of QTc prolongation by these medications is enhanced in the setting of renal failure, older age, baseline QTc prolongation and the concomitant use of other QT-prolonging medications in patients with severe COVID-19 infection. A better understanding of these factors associated with QTc prolongation is important for safe administration of these medications, especially in light of data showing low efficacy in COVID-19 treatment.

\section{References}


1. Gollob MH, COVID-19, Clinical Trials and QT-Prolonging Prophylactic Therapy in Healthy Subjects: First, Do No Harm, Journal of the American College of Cardiology (2020), doi: https://doi.org/10.1016/j.jacc.2020.05.008.

2. Mercuro NJ, Yen CF, Shim DJ, Maher TR, McCoy CM, Zimetbaum PJ, Gold HS. Risk of QT Interval Prolongation Associated With Use of Hydroxychloroquine With or Without Concomitant Azithromycin Among Hospitalized Patients Testing Positive for Coronavirus Disease 2019 (COVID-19). JAMA Cardiol. 2020 May 1: e201834. doi: 10.1001/jamacardio.2020.1834

3. Bessière F, Roccia H, Delinière A, Charrière R, Chevalier P, Argaud L, Cour M. Assessment of QT Intervals in a Case Series of Patients With Coronavirus Disease 2019 (COVID-19) Infection Treated With Hydroxychloroquine Alone or in Combination With Azithromycin in an Intensive Care Unit. JAMA Cardiol. 2020 May 1 : e201787. doi: 10.1001/jamacardio.2020.1787

4. Saleh M, Gabriels J, Chang D, Kim BS, Mansoor A, Mahmood E, Makker P, Ismail H, Goldner B, Willner J, Beldner S, Mitra R, John R, Chinitz J, Skipitaris N, Mountantonakis S, Epstein LM. The Effect of Chloroquine, Hydroxychloroquine and Azithromycin on the Corrected QT Interval in Patients With SARS-CoV-2 Infection. Circ Arrhythm Electrophysiol 2020 Apr 29. doi: 10.1161/CIRCEP.120.008662. PMID: 32347743 DOI: 10.1161/CIRCEP.120.008662

5. Rosenberg ES, Dufort EM, Udo T, Wilberschied LA, Kumar J, Tesoriero J, Weinberg P, Kirkwood J, Muse A, DeHovitz J, Blog DS, Hutton B, Holtgrave DR, Zucker HA. Association of Treatment With Hydroxychloroquine or Azithromycin With In-Hospital Mortality in Patients With COVID-19 in New York State. JAMA; 2020 May 11;e208630. doi: 10.1001/jama.2020.8630. Online ahead of print.

6. Drew BJ, Ackerman MJ, Funk M et al. Prevention of torsade de pointes in hospital settings: a scientific statement from the American Heart Association and the American College of Cardiology Foundation. Circulation 2010;121:1047-60.

Figure Legend

Figure 1. Telemetry rhythm strip demonstrating significant QTc prolongation during sinus rhythm followed by a self-terminating run of Torsades de Pointes.

Figure 1.

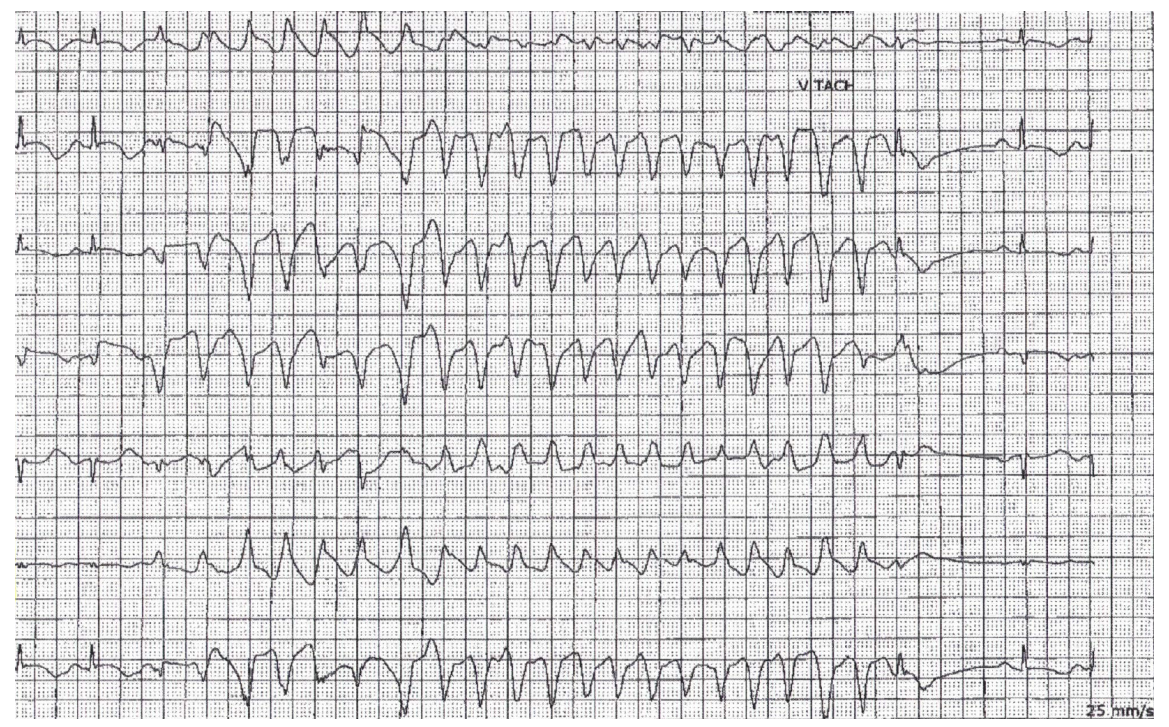

Table 1. Baseline Characteristics

\section{Hosted file}


image2.emf available at https://authorea.com/users/325952/articles/453900-incidenceand-determinants-of-qt-interval-prolongation-in-covid-19-patients-treated-withhydroxychloroquine-and-azithromycin

Table 2. Univariable and Multiple Logistic Regression Analysis

Hosted file

image3.emf available at https://authorea.com/users/325952/articles/453900-incidenceand-determinants-of-qt-interval-prolongation-in-covid-19-patients-treated-with-

hydroxychloroquine-and-azithromycin

$\mathrm{QTc}=$ corrected QT interval, $\mathrm{CRP}=\mathrm{C}$-Reactive Protein, $\mathrm{Tn}=$ Troponin $\mathrm{T}, \mathrm{K}=$ serum potassium, GFR $=$ glomerular filtration rate 